\title{
Serial Block-Face SEM of Brain Tissue Using Rapid Automated Preparation
}

Emily Benson ${ }^{1}$, Grahame Kidd ${ }^{1}$, Jay Campbell ${ }^{2}$ and Steven Goodman ${ }^{3}$

${ }^{1}$ Cleveland Clinic, Cleveland, Ohio, United States, ${ }^{2}$ University of Wisconsin-Madison, Madison, Wisconsin, United States, ${ }^{3}$ Microscopy Innovations, LLC, Marshfield, Wisconsin, United States

Serial block-face electron microscopy (SBEM) provides powerful 3D insight into neurological structures for mapping and quantification. While image acquisition is now largely computer controlled, SBEM sample preparation requires tedious circa week-long manual exchanges of toxic reagents, that are similar to but even more extensive and laborious than the chemical sample preparation required for transmission electron microscopy (TEM).

We report herein an SBEM protocol for neurological tissue where all reagent exchanges are automatically performed in one day using the programmable mPrep ASP-1000 Automated Specimen Processor (ASP). The ASP enables high-speed preparation of biological tissues with rapid and repeated fluid exchanges that accelerate reagent diffusion into specimens. This has been previously demonstrated for TEM [1]. For SBEM, a 4 to 5 day manual preparative protocol [2] was reduced to 7.5 hours of automated processing, followed by resin curing.

Adult rats were perfusion-fixed with cacodylate-buffered glutaraldehyde-paraformaldehyde. For ASP preparation, cortex specimens (1-3 mm) were oriented in mPrep/s capsules, loaded onto the ASP, and all preparative reagents were aliquoted into microwell plates on the ASP stage. The ASP executed the protocol by aspirating successive reagents into each capsule for the programmed time (Fig. 1, Table 1) with agitation provided by gentle flow reversals every few seconds. Epoxy infiltrated specimens in the capsules were then removed from the ASP and cured overnight at 60C. Manual preparation was performed in vials and cured in flat molds [2]. A ThermoFisher Teneo VolumeScope imaged blocks at $2.0 \mathrm{kV}, 0.1$ $\mathrm{nA}$ under high vacuum with the T1 BSE detector. Imaged volumes were $\sim 60$ x 60 um by 20 um from 70 nm sections.

Specimens prepared with the 7.5 hour ASP protocol (plus resin curing) and the 4-day manual protocol were compared from the same animal. Myelin staining in both indicated good reagent penetration since lipid-rich myelin is a dense target for metal staining and a barrier to diffusion. Synaptic vesicle clarity and discrimination of mitochondrial cristae against the dark mitochondrial matrix were also comparable. Neither ASP or manually-prepared specimens exhibited deficits characteristic of poor staining, dehydration, or infiltration (Fig. 1).

SBEM can reveal complex 3D neural architectures that are not apparent from single plane images. Figure 2 shows a cortical dendrite in a single plane image (not unlike a TEM section) and a 3D volume rendered reconstruction from the SBEM data, which reveals complex pre-synaptic features that are not apparent in single plane images. With ASP-enabled automation of SBEM preparation, the ability to image such complex 3D neurological (and other tissue) structures becomes more easily achieved with less effort and greater reproducibility. Additionally, since the ASP reduces reagent handling, and as it vents into or is contained in a fume hood, experimenter exposure to noxious reagents may also be reduced. 


\begin{tabular}{|c|c|c|}
\hline Reagent & $\begin{array}{c}\text { Manual } \\
\text { Time (min) }\end{array}$ & $\begin{array}{l}\text { ASP-1000 } \\
\text { Time (min) }\end{array}$ \\
\hline Karnovsky fix & Store $5 \mathrm{C}$ & Store $5 \mathrm{C}$ \\
\hline Buffer & 30 & 3 \\
\hline Tannic acid (some) & 15 & 15 \\
\hline OsO4 - KFeCN & 180 & 60 \\
\hline Water & 25 & 15 \\
\hline $1 \% \mathrm{TCH}$ & 60 & 60 \\
\hline Water & 25 & 3 \\
\hline $2 \% \mathrm{OsO} 4$ & 180 & 30 \\
\hline Water & 25 & 15 \\
\hline $2 \%$ Uranyl Acetate & 1,200 & 60 \\
\hline Water & 25 & 9 \\
\hline Lead Aspartate & 40 & 30 \\
\hline Water & 25 & 9 \\
\hline Graded ethanols & 105 & 53 \\
\hline Acetone & 30 & 9 \\
\hline Epoxy-acetones & 600 & 30 \\
\hline $100 \%$ epoxy & 90 & 30 \\
\hline Transfer tissue into molds & 45 & 0 \\
\hline Resin cure $60 \mathrm{C}$ & 2 days & Overnight \\
\hline $\begin{array}{c}\text { Protocol time } \\
\text { Hands-on labor time }\end{array}$ & $\begin{array}{l}4 \text { days } \\
2 \text { days }\end{array}$ & $\begin{array}{c}1 \text { day } \\
1 \mathrm{hr}\end{array}$ \\
\hline
\end{tabular}

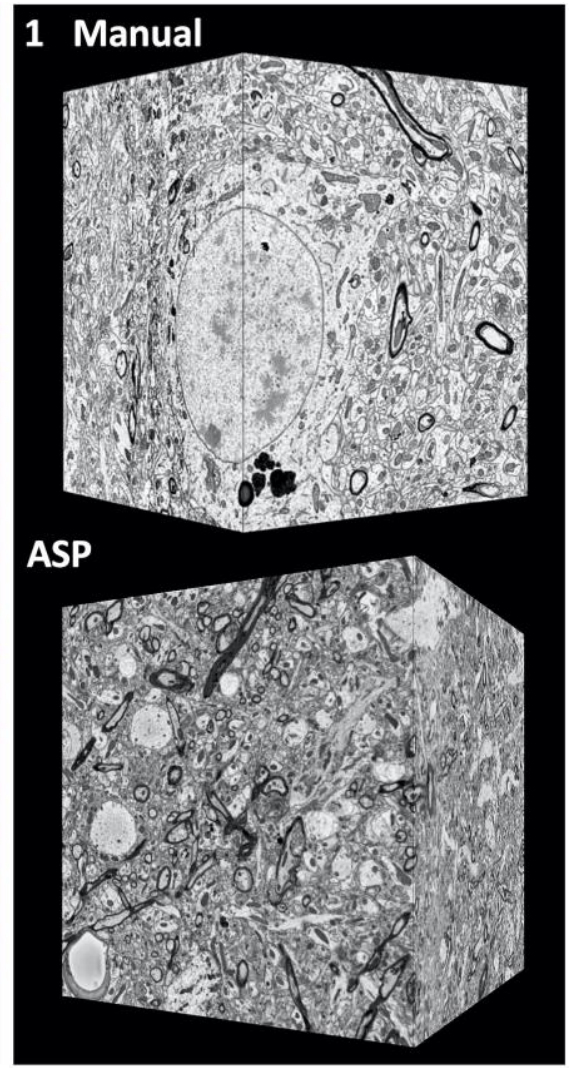

Figure 1. Table 1: Reagent protocol, times, and hands-on labor effort for manual and ASP preparation. Figure 1: Perspective projections of cortex prepared manually (top) and with ASP (bottom). $\sim 60 \mathrm{x} 60 \mathrm{x}$ $20 \mu \mathrm{m}$ deep, from $35070 \mathrm{~nm}$ thick slices. Images acquired in $25 \mathrm{hr}$ each.

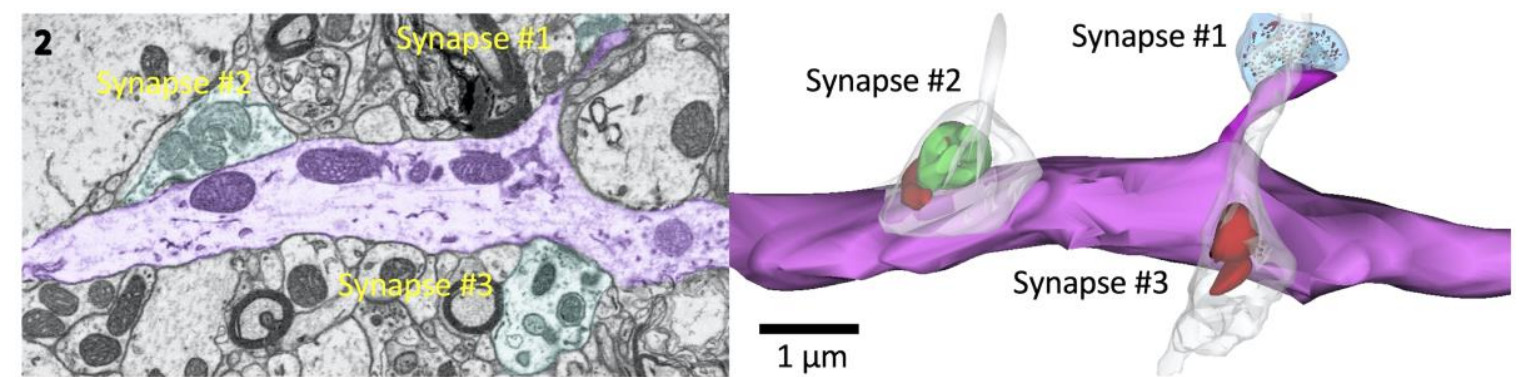

Figure 2. Cortical dendrite (magenta) single plane image (left) with 3 synapses and 3D volume rendered image (right). 3D rendering show complex architecture including synaptic vesicles in Synapse \#1, and mitochondria in Synapses \#2 and \#3.

\section{References}

[1] Strader TE, et al, Microsc. Microanal. 24 (2018) 1284.

[2] Deerinck, TJ, et al, Microscopy, (2010), 6. 